\title{
Dielectric Lens Antennas for 300-GHz Applications
}

\author{
Kimmo Rasilainen, Jiangcheng Chen, Markus Berg, and Aarno Pärssinen \\ Centre for Wireless Communications, University of Oulu, P. O. Box 8000, FI-90014 Oulu, Finland \\ kimmo.rasilainen@oulu.fi
}

\begin{abstract}
Implementation of proper wireless links at submillimetre-wave and terahertz (THz) frequencies requires high-gain antennas. This simulation-based study investigates the performance of dielectric lenses and their feed antennas at 300 GHz. Parametric studies and electromagnetic simulations are used to determine the general lens properties, and the use of waveguide and on-chip antenna feeds is considered. The results show that the studied lens and feed structures can provide good, directive radiation patterns also with beam scanning.
\end{abstract}

Index Terms-antenna, directivity, efficiency, lens, terahertz (THz), wireless communications.

\section{INTRODUCTION}

Wireless communications systems are developing at an unprecedented rate. Currently, fifth-generation (5G) networks and systems are being introduced [1], and the planning and visioning of solutions beyond $5 \mathrm{G}$ (known as $6 \mathrm{G}$ ) has already started (see, e.g., [2]). All of these wireless systems are facing ever-increasing technical requirements in terms of, e.g., data rate, latency, and the number of simultaneous users.

Considering these requirements and spectral availability, the focus of the research is switching towards millimetre and submillimetre-wave frequencies. As the frequency increases, the path loss across a certain link distance also significantly increases and begins to limit the obtainable communication distance. The path loss can be compensated for by using more directive antennas (e.g., larger antenna arrays and/or lenses).

Generally speaking, two main approaches can be used to improve the data rate: better spectral efficiency and broader bandwidth. The former approach involves the use of higherorder modulation schemes and/or multi-antenna (MIMO) techniques. When targeting for data rates around and beyond several tens of $\mathrm{Gb} / \mathrm{s}$, achieving this by means of spectral efficiency becomes technically very challenging [3]. From a bandwidth point of view, the $300-\mathrm{GHz}$ and beyond frequency range has gained significant interest, as it features large sections of contiguous, non-regulated spectrum which can be a possible candidate for ultra-high data rate communications.

With higher frequencies, the physical size corresponding to a certain electrical size decreases and, as a result, the individual antennas become smaller. However, the electronics needed to drive the antennas does not shrink at the same rate, and therefore the overall size of the antenna/front-end system can become limited by the active circuits. For the electronics, higher frequencies present new challenges in terms of, e.g., generating the required power levels or choosing suitable semiconductor fabrication technologies [4], [5]. One possible

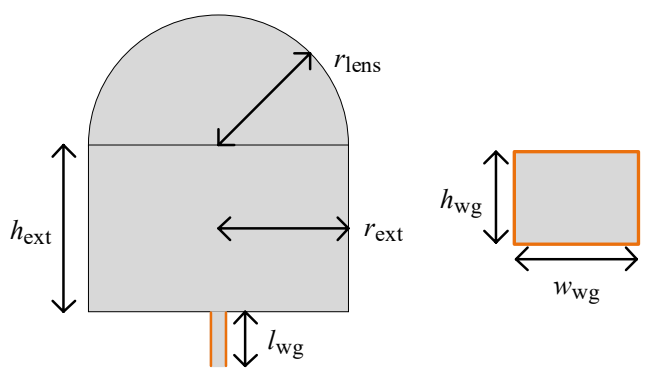

Fig. 1. Schematic illustration of the investigated lens antenna geometry and waveguide feed. The drawing is not entirely to scale.

technology is silicon germanium ( $\mathrm{SiGe}$ ) based heterojunction bipolar transistor (HBT) technology used in, e.g., [6], [7].

This simulation-based study investigates the design and performance of silicon lens antennas and their feed structures for $300-\mathrm{GHz}$ telecommunications applications. The aim is to understand the principal possibilities and challenges associated with the different solutions. Simulations are carried out using the CST Microwave Studio software.

\section{Parametric Studies on the Lens Performance}

The first step is to consider the fundamental properties of the lens through parametric studies on its dimensions. In this part, an extended hemispherical lens with a waveguide feed is investigated, and Fig. 1 illustrates the studied geometry. The extended hemispherical shape is more straightforward to implement in practice than an ideal ellipsoidal lens shape [8], and placing a feed antenna in its focus will result in a far-field pattern whose main-beam properties are determined by the lens aperture [9]. The material of the lens is silicon $\left(\varepsilon_{\mathrm{r}}=11.9\right.$, $\tan \delta=1.2 \cdot 10^{-6}$ at $300 \mathrm{GHz}$ ).

The rectangular feed waveguide is a dielectric-filled WR3 waveguide $(220-325 \mathrm{GHz})$, and the filling material is the same as that of the lens. Consequently, the aperture size of the waveguide is scaled down to account for the effect of the dielectric. In this work, $w_{\mathrm{wg}}=0.7 \mathrm{~mm}, h_{\mathrm{wg}}=0.362 \mathrm{~mm}$, and $l_{\mathrm{wg}}=1 \mathrm{~mm}$. The purpose of the filling is to reduce the reflections at the lens-to-waveguide interface. As an alternative to filling, a small air cavity could be carved into the lens extension, but the optimal cavity depth is wavelength-dependent and may result in some bandwidth limitations [10].

\section{A. Effect of Extension Height and Lens Diameter}

In the geometry of Fig. 1, two dimensions are considered for the parametric analysis: lens diameter $\left(d_{\text {lens }}\right)$ and height of the 


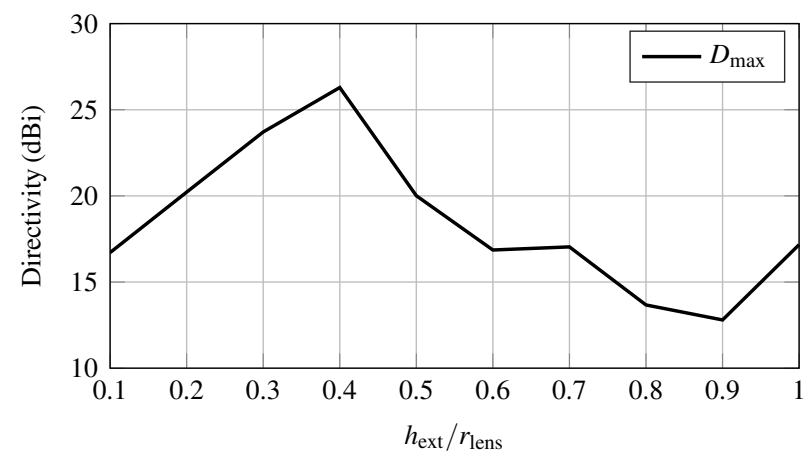

(a)

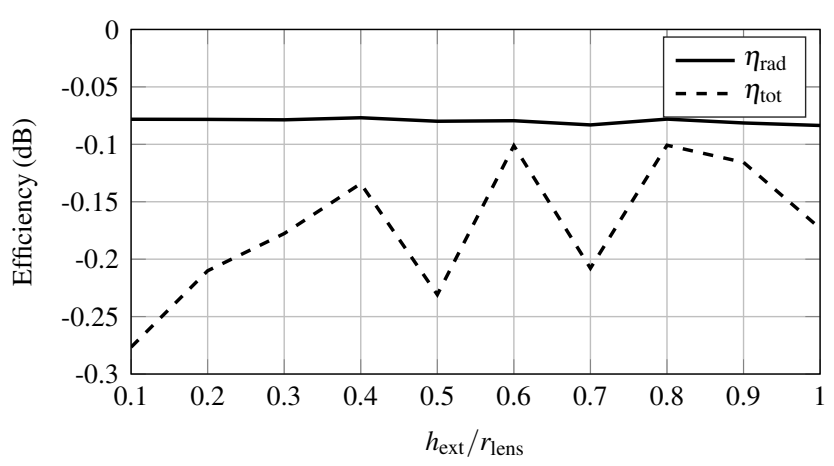

(b)

Fig. 2. Simulated lens (a) maximum directivity $\left(D_{\max }\right)$ and (b) efficiency as a function of extension height. A waveguide feed is used, and $d_{\text {lens }}=10 \mathrm{~mm}$

cylindrical extension $\left(h_{\text {ext }}\right)$. Their effect on the lens directivity and (total) efficiency is considered, and the frequency of interest is $300 \mathrm{GHz}$. The diameter of the lens and extension are the same, and the properties of the feed structure are not modified in this part. Thus, it can be assumed that most of the observed effects are caused by the parameters under study.

Fig. 2(a) shows the simulated peak lens directivity and efficiency of a lens whose diameter is $10 \mathrm{~mm}$ when the height of the cylindrical extension is changed proportional to the lens radius $\left(0.1 \leq h_{\text {ext }} / r_{\text {lens }} \leq 1\right)$. The curve shows that the directivity peaks at around $h_{\text {ext }} / r_{\text {lens }}=0.4$, which agrees very well with the reported optimal extension height of $h_{\text {ext }} / r_{\text {lens }} \approx 0.39$ for a Si lens [11], [12].

With the currently assumed material parameters, the radiation efficiency of the lens-waveguide system is very good, as can be seen in Fig. 2(b). Changes in the total efficiency performance relate to variations in the matching. This effect may be a result of changes in internal reflections in the lens caused by the varying extension height.

The second parameter to consider in this study is the lens diameter. Based on the above results, an extension height of $0.39 r_{\text {lens }}$ is used, and the same value is also applied to all subsequent studies described in this paper. The diameter of the lens is varied from 10 to $40 \mathrm{~mm}$ with $5-\mathrm{mm}$ increments, and the same waveguide feed is used as before. Structural symmetry is applied to reduce the computational time.

Fig. 3 depicts the dependence of the lens directivity and

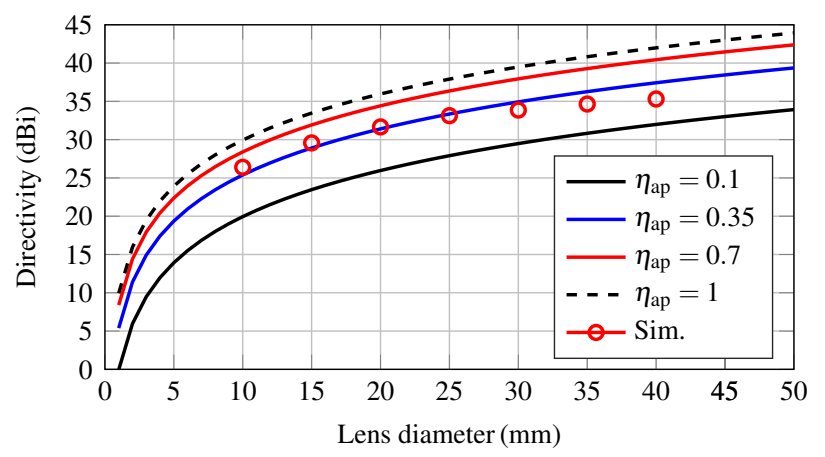

(a)

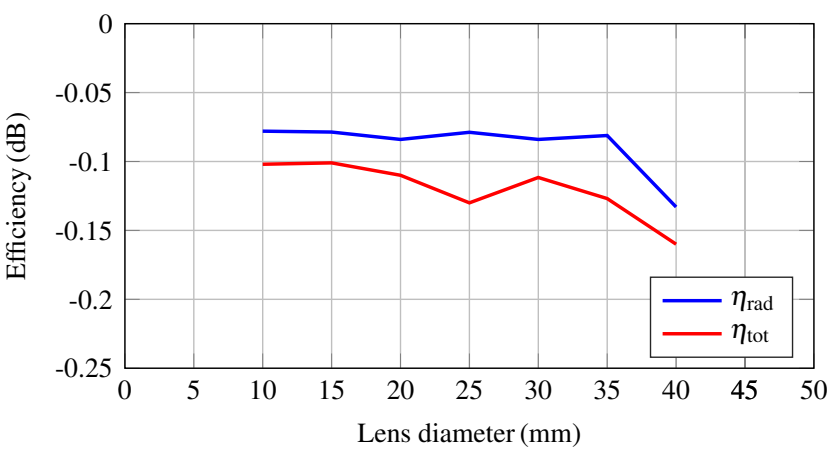

(b)

Fig. 3. Simulated lens (a) maximum directivity $\left(D_{\max }\right)$ and (b) efficiency as a function of lens diameter. A waveguide feed is used, and $h_{\text {ext }} / r_{\text {lens }}=0.39$.

efficiency on the diameter. In addition to the simulated directivity, the figure shows the theoretical directivity for a circular aperture (in this case, the lens) for different values of aperture efficiency $\left(\eta_{\mathrm{ap}}\right)$. The directivity is calculated using Eq. (1):

$$
D(\mathrm{~dB})=10 \lg \left(4 \pi \eta_{\mathrm{ap}} A / \lambda^{2}\right),
$$

in which $\eta_{\text {ap }}$ is the aperture efficiency and $A$ is the aperture area. Ideally, $\eta_{\text {ap }}=1$ but typical values are, e.g., 0.35 or 0.7 .

As can be seen in Fig. 3(a), the simulated directivity follows the general trend of the theoretical results, even though the simulated values start to saturate to approximately $35 \mathrm{dBi}$ with larger lenses. This behaviour might be related to the current waveguide feed, which is assumed to have a constant aperture. Thus, its focal point may be more focal-plane like (equal to the aperture size) with the chosen $h_{\text {ext }}$, which reduces the directivity. It may be possible to compensate for this drop in directivity by optimising the $h_{\text {ext }}$ value for the different lenses (especially the larger ones), but further studies are needed.

With increasing lens diameter, the efficiency gradually decreases (mainly due to increasing material losses). In practical implementations, the absolute drop in efficiency depends on the properties of the particular Si material used but based on the current results, the relative drop is not very significant.

\section{B. Frequency Dependence of the Lens Properties}

The previous results have presented how the directivity and efficiency behave as a function of lens dimensions, but they 


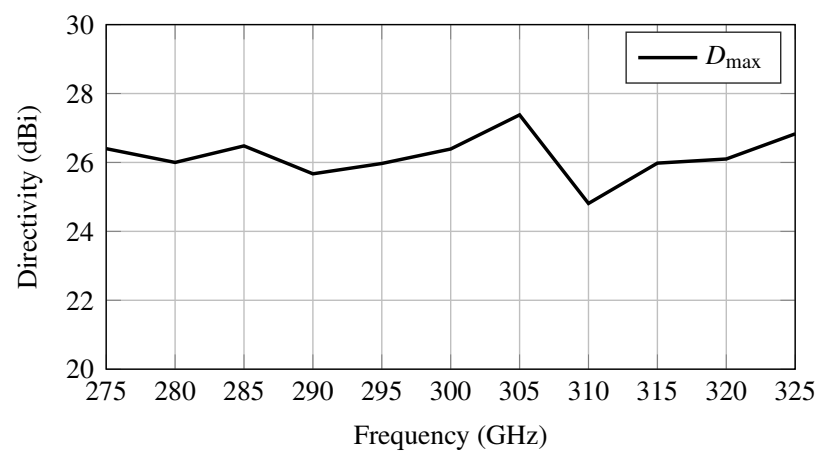

(a)

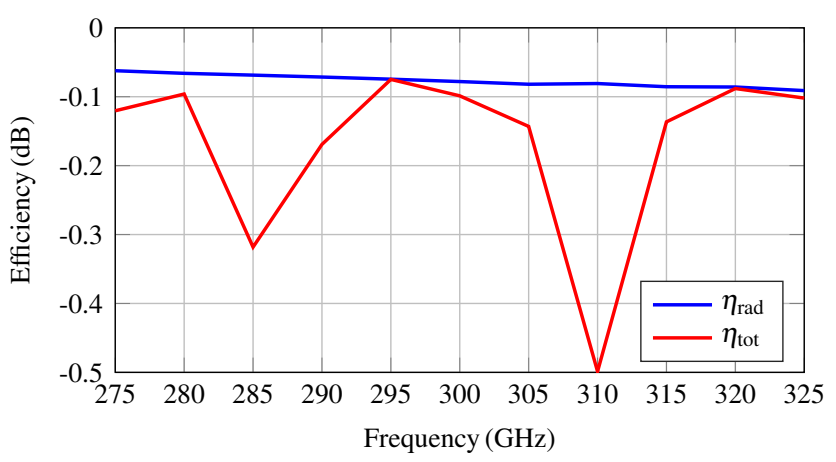

(b)

Fig. 4. Simulated lens (a) maximum directivity $\left(D_{\max }\right)$ and (b) efficiency as a function of frequency when $d_{\text {lens }}=10 \mathrm{~mm}$ and $h_{\mathrm{ext}} / r_{\text {lens }}=0.39$.

do not show how these parameters change with frequency. As a way of describing this, Fig. 4 illustrates the frequency dependence of the directivity and efficiency for a fixed lens size $\left(d_{\text {lens }}=10 \mathrm{~mm}, h_{\text {ext }} / r_{\text {lens }}=0.39\right)$.

The curves of Fig. 4 show that the directivity somewhat fluctuates with frequency, but the average peak directivity across the studied frequency range is approximately $26 \mathrm{dBi}$, which is also in accordance with the result of Fig. 3(a). Obtained efficiency values are generally in the same range as the dimension-dependent results shown above.

\section{Combined EFfect of Lens And FeED AntennA}

For various applications, such as multi-pixel $\mathrm{THz}$ detectors, waveguide-based feeds may not be suitable in practice. Rather, the required antennas need to be implemented as planar arrays whose size and configuration are application-specific. Therefore, the next step is to consider the performance of the lens with a more representative on-chip feed antenna.

This study is performed for the previously-used 10-mm lens which is computationally more efficient to analyse. Additionally, it serves as a proof-of-concept model since the current work does not consider any particular link requirements (range, data rate, beamwidth etc.). Apart from the feed antenna, the general configuration is similar to that of Fig. 1.

\section{A. Properties of the Feed Antenna}

The on-chip feed antenna used together with the lens is a dipole with a reflector, as shown in Fig. 5. Two different configurations are studied: an individual feed antenna, and a linear $1 \times 4$ antenna array. The basic stand-alone antenna design has been adapted (with slight modifications) from [13], but the implemented array design and the use of these antennas with the lens represent original research by the authors.

In both configurations, the substrate on the antenna chip consists of two layers: a $6-\mu \mathrm{m}$ thick benzocyclobutediene (BCB) layer, beneath which is a layer of indium phosphide (InP) whose thickness is $50 \mu \mathrm{m}$. The material parameters for these layers at $300 \mathrm{GHz}$ are assumed to be $\varepsilon_{\mathrm{r}}=2.5$ and $\tan \delta=0.005$ for $\mathrm{BCB}$ and $\varepsilon_{\mathrm{r}}=12.5$ and $\tan \delta=0.003$ for InP [13]. The dipole and reflector are modelled as a $2-\mu \mathrm{m}$ thick gold layer. In the array, the individual antenna chips are placed on a block of FR-4 $\left(\varepsilon_{\mathrm{r}}=4.3, \tan \delta=0.025\right)$ which is used to model a printed circuit board (PCB).

Fig. 6 shows the simulated $S$-parameters of the single dipole and the $1 \times 4$ array both in free space and in the presence of a semi-infinite Si-filled half space. The latter case describes the performance of the antenna in the presence of the lens, whose size is electrically very large compared to the antenna chip. As can be seen in the curves, including the dielectric affects both the matching level and impedance bandwidth of the antenna, but the performance at the targeted $300-\mathrm{GHz}$ frequency remains good both for the single antenna and for the array. The distance between adjacent antenna elements in the array $(500 \mu \mathrm{m}$ centre-to-centre) has been chosen in a way that the matching level at $300 \mathrm{GHz}$ is as good as possible.

\section{B. Lens Performance with Single-Element and Array Feeds}

The operation of the lens is investigated using both a single feed antenna and the array configuration in different scenarios to study the phased-array/beamscanning properties. Three different excitation scenarios are considered with the array: 1) simultaneous excitation of two centremost elements $(1 \times 2$ array), 2) simultaneous excitation of all four elements, and 3) exciting one element of the array at a time. In the first two phased-array type scenarios, the beamsteering properties by tuning the amplitude and phase of the input signals are beyond the scope of the present work. The third case is an example of beam switching, whereby selecting the desired active feed element a different main-beam direction is obtained.

Fig. 7 illustrates the peak directivity and efficiency characteristics for the single feed and simultaneous excitation cases. It can be seen that the directivity decreases when increasing the number of simultaneously excited elements. This effect relates to the lateral displacement $\left(l_{\text {dis }}\right)$ of the individual antennas from the lens centre/focus, which results in their beams pointing in different directions [14]. Due to the different radiation directions, the beams of adjacent elements can be out of phase in such a way that nulls begin to appear in the pattern. A number of peaks matching the number of excited antennas is seen. The issue of beam misalignment can be reduced by increasing the diameter of the lens, as the resulting steering angle relates to the relative displacement $l_{\text {dis }} / r_{\text {lens }}$ [14], [15].

For a more detailed look at the effect of the feed antenna on the radiation pattern shape, Fig. 8 shows two-dimensional 


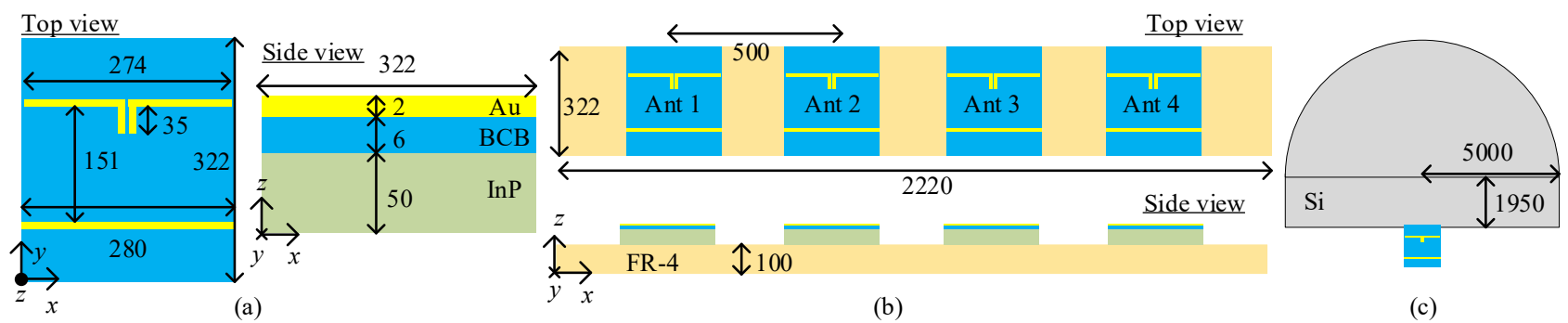

Fig. 5. Illustration of the (a) dipole antenna and (b) antenna array used to feed the lens, and (c) lens and antenna chip configuration. The basic design for the dipole is adapted from [13]. The drawing is not entirely to scale. All dimensions are in $\mu \mathrm{m}$.

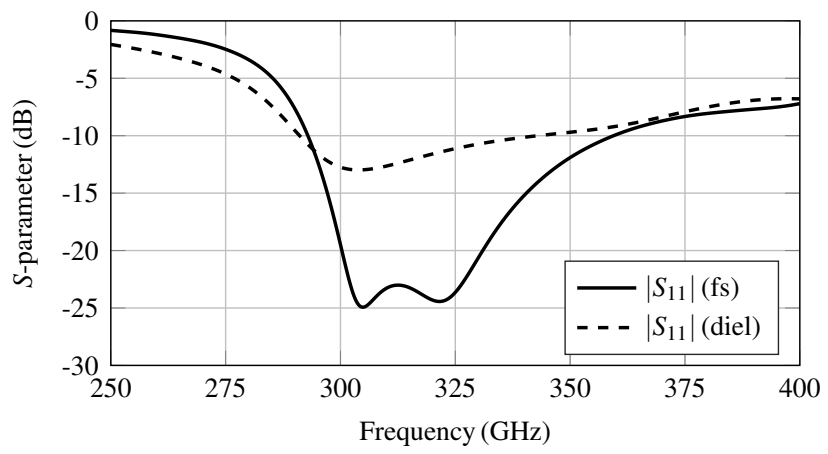

(a)

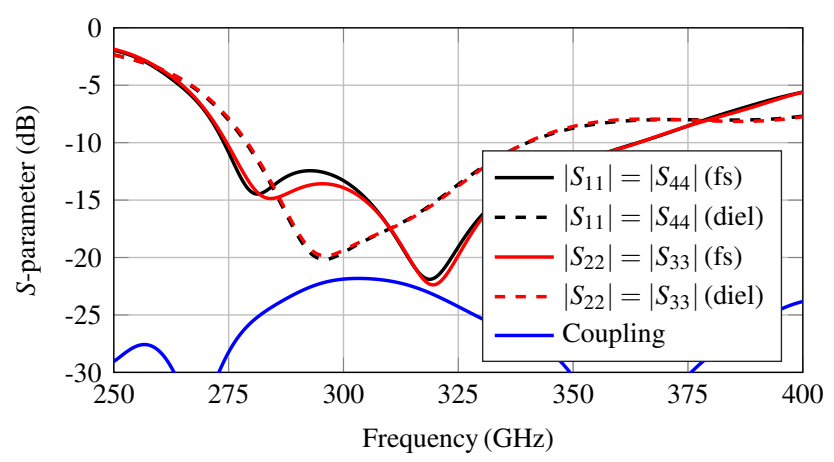

(b)

Fig. 6. Simulated feed antenna $S$-parameters for the (a) single dipole and (b) $1 \times 4$ array in free space (fs) and with a semi-infinite Si half space (diel). Coupling curve: worst-case mutual coupling in the antenna array.

$E$-plane cuts of the obtained far-field patterns at $300 \mathrm{GHz}$ with the different feed configurations. It can be seen that the peak directivity decreases and the main beam becomes broader when we increase the number of simultaneously active elements. The lens creates an image of the feed antennas, and as result, the radiation spreads over a broader angle [16].

With the $1 \times 4$ array, the outermost peaks start to drop down in magnitude compared to the centremost ones. This is caused by internal reflections in the lens-to-air interface, which relate to the contrast in material parameters at the interface (refractive index $n \propto \varepsilon_{\mathrm{r}}$ ) [17]. By applying a matching layer or anti-reflective coating on the lens surface, the effect can be reduced and the peaks in the pattern made symmetrical [18].

In the beam-switching case, the main beam pattern shape

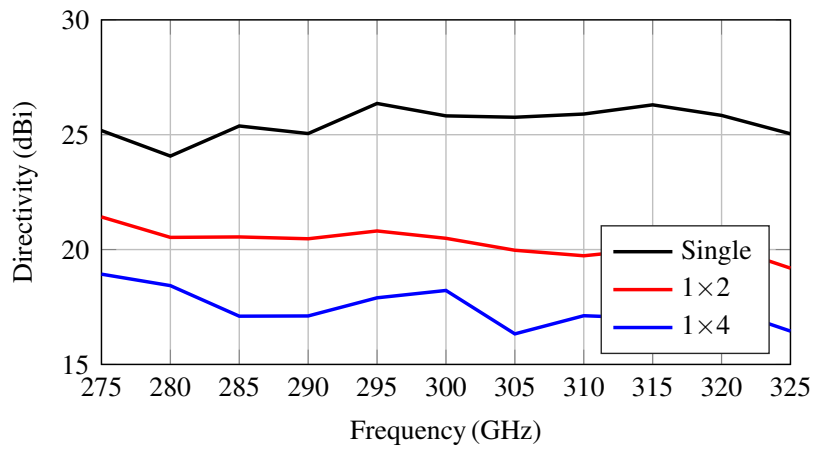

(a)
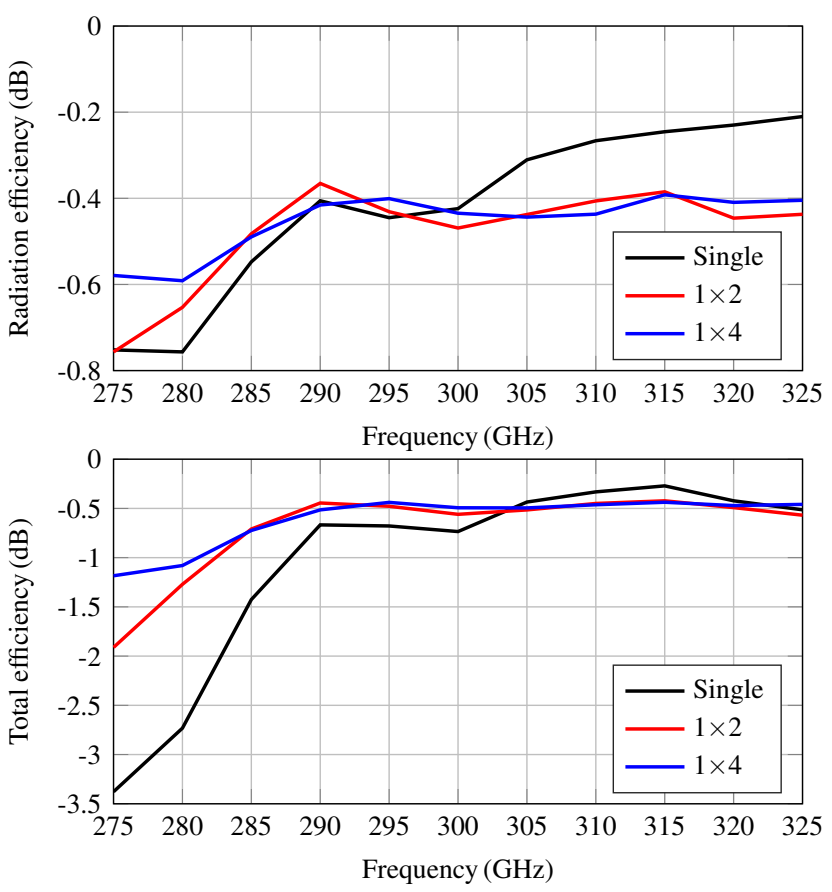

(b)

Fig. 7. Simulated lens (a) maximum directivity $\left(D_{\max }\right)$ and (b) efficiency with different feed configurations (single dipole, $1 \times 2$ array, and $1 \times 4$ array).

remains good, even though the magnitude slightly drops from the boresight direction. The single dipole is placed at the focus of the lens whereas the array with an even number of elements is aligned according to its geometric centre, which causes 


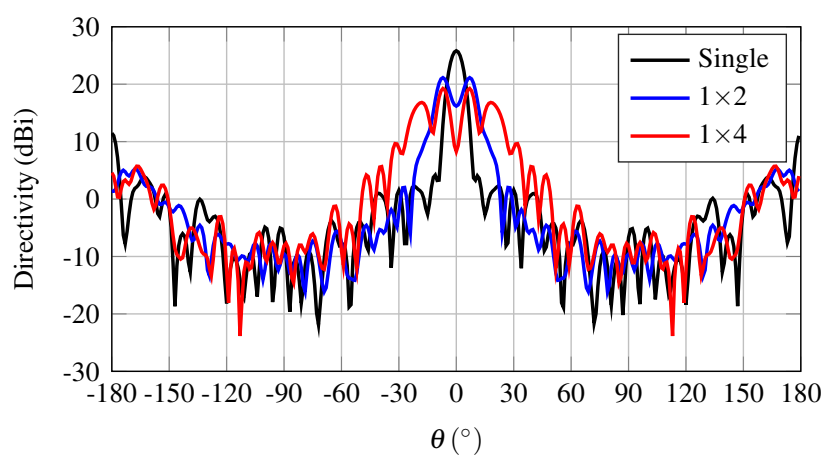

(a)

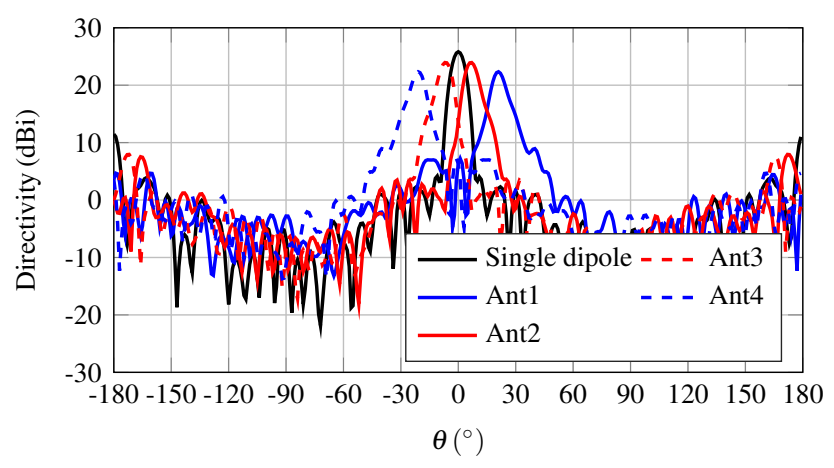

(b)

Fig. 8. Two-dimensional cuts of the far-field pattern ( $E$-plane) of the lens antenna (single-antenna and array feeds) with (a) simultaneous element excitation and (b) beam switching (one element of the array active at a time). The locations of Ant1-Ant 4 in the array follow the notation of Fig. 5.

slight differences in the main beam magnitudes compared to the single-feed case. Beam switching performance can be improved by optimising the feed array (size, element spacing etc.), but this aspect was not considered in this work.

In addition to affecting the directive properties of the lens antenna system, the propagation of the rays within and away from the lens also contributes to the overall efficiency. Without an anti-reflective coating or absorbing material around the lens, part of the radiation escapes from the extension part (spillover loss). Also the backlobe of the feed antenna pattern, which is more pronounced with the antennas of Fig. 5, carries energy towards unwanted directions. Additional analysis on this aspect, e.g., using ray-tracing based techniques would be needed for an improved understanding on this part.

\section{CONCLUSION}

This work has investigated how the performance of an integrated $\mathrm{THz}$ lens antenna depends on the lens dimensions and the properties of the feed antennas. Both waveguide feeds and on-chip antennas are used. Parametric studies and electromagnetic simulations have identified suitable dimensions for implementing a well-performing Si lens. The current lens and feed antenna configuration provides a directivity of $25 \mathrm{dBi}$, which can be adjusted to meet particular requirements by changing the lens size. Results of this study provide useful guidelines for implementing high-performance antenna solutions for $\mathrm{THz}$ wireless communications. Future and ongoing work includes lens fabrication and designing integrated transmitter/receiver circuits to perform experimental verifications of the studied lens antenna system.

\section{ACKNOWLEDGEMENT}

This work was supported by the Academy of Finland 6Genesis Flagship Programme (grant no. 318927).

\section{REFERENCES}

[1] J. G. Andrews et al., "What will 5G be?" IEEE J. Sel. Areas Commun., vol. 32, no. 6, pp. 1065-1082, Jun. 2014.

[2] M. Latva-aho and K. Leppänen (eds.), "Key drivers and research challenges for 6G ubiquitous wireless intelligence," White Paper, 6G Flagship, University of Oulu, Finland, Sep. 2019.

[3] T. Kürner and S. Priebe, "Towards THz communications - status in research, standardization and regulation," J. Infrared Milli Terahz, Waves, vol. 35, no. 1, pp. 53-62, Jan. 2014.

[4] Z. Chen et al., "A survey on terahertz communications," China Commun., vol. 16, no. 2, pp. 1-35, Feb. 2019.

[5] L. Bariah et al., "A prospective look: Key enabling technologies, applications and open research topics in $6 \mathrm{G}$ networks," IEEE Access, vol. 8, pp. 174792-174820, 2020.

[6] J. Grzyb, K. Statnikov, N. Sarmah, B. Heinemann, and U. R. Pfeiffer, "A 210-270-GHz circularly polarized FMCW radar with a single-lenscoupled SiGe HBT chip," IEEE Trans. THz Sci. Technol., vol. 6, no. 6 , pp. 771-783, Nov. 2016.

[7] P. Rodríguez-Vázquez, J. Grzyb, B. Heinemann, and U. R. Pfeiffer, "A QPSK 110-Gb/s polarization-diversity MIMO wireless link with a 220 $255 \mathrm{GHz}$ tunable LO in a SiGe HBT technology," IEEE Trans. Microw. Theory Techn., vol. 68, no. 9, pp. 3834-3851, Sep. 2020.

[8] G. Carpintero, L. E. García Muñoz, H. L. Hartnagel, S. Preu, and A. V. Räisänen (eds.), Semiconductor Terahertz Technology: Devices and Systems at Room Temperature Operation, John Wiley \& Sons, 2015.

[9] K.-C. Huang and D. J. Edwards, Millimetre Wave Antennas for Gigabit Wireless Communications: A Practical Guide to Design and Analysis in a System Context, John Wiley \& Sons, 2008.

[10] K. Konstantinidis et al., "Low-THz dielectric lens antenna with integrated waveguide feed," IEEE Trans. THz Sci. Technol., vol. 7, no. 5, pp. 572-581, Sep. 2017.

[11] N. Llombart, G. Chattopadhyay, A. Skalare, and I. Mehdi, "Novel terahertz antenna based on a silicon lens fed by a leaky wave enhanced waveguide," IEEE Trans. Antennas Propag., vol. 59, no. 6, pp. 2160 2168, Jun. 2011.

[12] A. Karttunen, J. Ala-Laurinaho, R. Sauleau, and A. V. Räisänen, "Extended hemispherical integrated lens antenna with feeds on a spherical surface," Proc. 2013 7th Eur. Conf. Antennas Propag. (EuCAP), Gothenburg, Sweden, Apr. 2013, pp. 2539-2543.

[13] H. Vettikalladi, W. T. Sethi, M. A. Alkanhal, and M. Himdi, "SubTHz dipole antenna for future 5G wireless communication," Proc. 2019 2nd Int. Conf. Computer Applications \& Information Security (ICCAIS), Riyadh, Saudi Arabia, May 2019, 4 p.

[14] H. Jalili and O. Momeni, "A 0.46-THz 25-element scalable and wideband radiator array with optimized lens integration in 65-nm CMOS," IEEE J. Solid-State Circuits, vol. 55, no. 9, pp. 2387-2400, Sep. 2020.

[15] D. F. Filipovic, G. P. Gauthier, S. Raman, and G. M. Rebeiz, "Off-axis properties of silicon and quartz dielectric lens antennas," IEEE Trans. Antennas Propag., vol. 45, no. 5, pp. 760-766, May 1997.

[16] G. Ghazi, P. Sadri-Moshkenani, and R. Safian, "Array optimization for maximum realized gain in terahertz antenna with lens," Proc. 2019 IEEE Int. Symp. Antennas Propag. (AP-S), Atlanta, GA, USA, Jul. 2019, pp. 125-126.

[17] M. J. M van der Vorst, P. J. I. de Maagt, and M. H. A. J. Herben, "Effect of internal reflections on the radiation properties and input admittance of integrated lens antennas," IEEE Trans. Microw. Theory Techn., vol. 47, no. 9, pp. 1696-1704, Sep. 1999.

[18] N. T. Nguyen, R. Sauleau, M. Ettorre, and L. Le Coq, "Focal array fed dielectric lenses: An attractive solution for beam reconfiguration at millimeter waves," IEEE Trans. Antennas Propag., vol. 59, no. 6, pp. 2152-2159, Jun. 2011. 